Relations industrielles

Industrial Relations

\title{
The Family, William J. Goode, Foundations of Modern Sociology Series, Prentice-Hall, Inc., Englewood Cliffs, New Jersey. 1965. 120 pages.
}

\section{Ronald Pleau}

\section{Volume 21, numéro 2, 1966}

URI : https://id.erudit.org/iderudit/027686ar

DOI : https://doi.org/10.7202/027686ar

Aller au sommaire du numéro

\section{Éditeur(s)}

Département des relations industrielles de l'Université Laval

ISSN

0034-379X (imprimé)

1703-8138 (numérique)

Découvrir la revue

Citer ce compte rendu

Pleau, R. (1966). Compte rendu de [The Family, William J. Goode, Foundations of Modern Sociology Series, Prentice-Hall, Inc., Englewood Cliffs, New Jersey. 1965. 120 pages.] Relations industrielles / Industrial Relations, 21(2), 302-303. https://doi.org/10.7202/027686ar

Tous droits réservés (C Département des relations industrielles de l'Université Laval, 1966
Ce document est protégé par la loi sur le droit d'auteur. L’utilisation des services d'Érudit (y compris la reproduction) est assujettie à sa politique d'utilisation que vous pouvez consulter en ligne.

https://apropos.erudit.org/fr/usagers/politique-dutilisation/ 
Jusqu'ici le législateur donne une protection au brevet d'invention, mais non au secret technique. Et si un tel système a pu être établi pour le premier, on peut aussi le faire pour le second, en établissant un document de référence (attestant la possession personnelle) en termes de brevet.

\section{Fernand Blais}

The Rule of Law in European Integration, Stuart A. Scheingold, McGill University Press, Montréal, 1965. 331 pages.

Parmi les institutions dont nous entendons le plus parler dans l'économie européenne moderne, nous remarquons la Communauté du charbon et de l'acier (le plan Schuman), le Marché commun et l'Euratom. Or, pour que les états membres de ces institutions qui se sentent lésés dans leurs droits et qui ont des griefs à formuler à l'endroit des systèmes puissent avoir certains recours, il a fallu instituer également une cour de justice pour l'audition de ces cos.

Ainsi dans cet ouvrage l'auteur examine le rôle de cette cour et établit la croissance de son approche fonctionnelle vis-à-vis les cas économico-juridiques qui lui sont présentés. II s'en tient ici à ceux formulés dans le cadre du plan Schuman lesquels sont d'ailleurs semblables aux autres et à ceux de l'intégration régionale en général. Ces cas constituent en quelque sorte une histoire judiciaire de cette institution et fournissent un excellent terrain d'étude pour une analyse.

Scheingold essaie de voir jusqu'à quel point après dix ans d'application du système de la communauté cette cour de justice s'est ajustée avec succès à son environnement et si cette dernière a apporté une contribution véritable au succès des Communautés mentionnées.

Selon les termes de l'auteur lui-même le but de cet exposé est de renseigner sur la relation réciproque qui existe entre les aspects légal et politique de l'intégration européenne.

Les deux premiers chapitres de cette étude soulignent la tension qui existe entre les rôles politiques et judiciaires de la cour de justice. Les chapitres suivants serviront à justifier l'argument qui veut que la cour oit adopté des techniques fonctionnelles et à démontrer le succès des méthodes fonctionnelles pour imposer des règles normatives au processus d'intégration.
Mais pour comprendre complètement la contribution du fonctionalisme, l'auteur voit la nécessité d'un côté, d'explorer ses limitations et, d'un autre côté, d'établir la faiblesse des méthodes olternatives adoptées par cette cour. C'est à cette tâche qu'il se consacre dans les deux chapitres suivants de la partie II.

Lo partie III traitera de l'évolution de la technique fonctionnelle et les IVe et $\mathrm{Ve}$ parties, de ses limitations, et plus spécialement des problèmes d'intégration partielle et de changement politique. II termine enfin sur une série de conclusions des plus intéressantes.

Le tout constitue donc une analyse des problèmes de conflits entre la recherche de la stabilité et le processus dynamique de I'intégration et de l'écart entre les institutions nationales juridiques et politiques.

\section{Ronald Pleau}

The Family, William J. Goode, Foundations of Modern Sociology Series, Prentice-Hall, Inc., Englewood Cliffs, New Jersey. 1965. 120 pages

II sera de tout temps nécessaire de traiter des relations familiales et d'insister sur les résultats et découvertes de la théorie sociologique à ce sujet.

Ainsi par exemple en étudiant les relations entre les systèmes de famille et la structure sociale plus vaste, nous pouvons dire que nous ne devons plus considérer la famille comme un simple ensemble de variables dépendantes expliquées par les \& forces économiques » seules. II faut chercher les relations entre les variables centrales et s'efforcer d'explorer la structure sociale plus vaste.

Quant au problème des valeurs, nous pouvons dire que l'analyse que contient ce volume n'en fait pas une évaluation mais que la sociologie comme science peut examiner de temps en temps certains modes alternatifs de vivre.

L'cuteur divise son oeurre en dix courts chapitres et considère la famille et les systèmes de relations familiales sous des aspects très variés bien que très loggiquement relics entre eux.

Toutes les sociétés ne conçoivent pas la famille de la même façon: certaines ont même des préjugés ou sujet de cette dernière. Mais quoiqu'il en soit, la famille restera toujours une institution unique qui 
peut être étudiée avec une approche sociologique. Bref elle est un élément de la structure sociale (chap. 1).

Cet élément de la structure sociale a des bases qu'on qualifie de biologiques. II sera donc nécessaire à l'auteur d'élaborer un second chapitre pour traiter de terminologie et de définitions, de facteurs culturels, du facteur sexuel et du lien entre le biologique et le culturel pour nous faire saisir en quoi consiste ces bases.

Puis après avoir discuté de légitimité et d'illégitimité par l'élaboration de quelques paragraphes sur le rôle clé de la socialisation, sur les normes sociales permettant de déterminer les types d'illégitimité et du contrôle de cette dernière, le responsable de cet exposé nous entretient sur quelques notions en rapport ovec le mariage.

Poursuivant dans le même sens on retrouve ensuite les implications des différents types de ménage que nous pouvons rencontrer, leur incidence, le type indien de la famille conjointe, etc. D'autres groupements organisés de descendance font I'objet d'un chapitre distinct de même que le rôle des relations entre les époux et les parents dans la famille et la société.

Un point intéressant qu'il faut noter est le rôle de la famille dans la stratification sociale.

Enfin la dissolution de l'institution familiale a des causes et des implications qui sont étudiées ici.

Bref la famille n'est pas une entité immuable et certains facteurs viennent parfois changer la face des modèles familiaux que nous sommes habitués de rencontrer et parmi ceux-ci un des plus importants est I'industrialisation; ce qui prouve que nous ne devons jamais perdre de vue les nombreuses relations entre les variables familiales et les autres variables de la société

Et comme le mentionne Goode « la vérification constante des hypothèses sur la façon dont le comportement de la famille est modelé nous aidera d̀ développer une conception plus précise au sujet du passé et de l'ovenir *

\section{Ronald Pleau}

Guide pour la prévention et la suppression des poussières dans les mines, les galeries et les carrières, Bureau International du Travail, Genève, 1965, 455 pages.
Ceux qui s'intéresesnt au monde du travail connaissent bien les intéressantes et précieuses publications du Bureau international du travail. Point n'est nécessaire de les vanter. Leurs antécédants parlent par eux-mêmes. Contentons-nous donc d'en décrire le contenu.

La sécurité et I'hygiène industrielles sont des sujets toujours d'actualité et une fois de plus nous retrouvons dans la matière qui nous est présentée dans cet ouvrage des conseils très utiles qui en plus de s'appliquer universellement demeurent d'une simplicité déconcertante.

Cette étude, destinée aux entreprises minières surtout, nous apporte quelques solutions pour la suppression des poussières, causes de tant de maladies. Ces solutions pourraient être mises en application avec avantage dans plusieurs milieux car comme on le mentionne d'ailleurs la société industrielle n'est pas très avancée dans ce domaine.

Le but que poursuivent les auteurs est "d'étudier les opérations d'exploitation ou de creusement au cours desquelles l'atmosphère risque de se charger de poussières et indiquer certains moyens de déterminer l'empoussièrement et d'en abaisser la concentration ».

Ainsi après avoir énuméré et expliqué les effets pathologiques des poussières minières, les compositeurs traitent des diverses sortes d'alimentation en eau des mines laquelle peut servir ensuite comme un des moyens de faire disparaître les poussières dangereuses.

$\mathrm{Ce}$ sont les systèmes d'aérage, de captage et de filtration des poussières au fond de la mine qui constitue l'objet des chapitres suivants avant de passer à la lutte contre les poussières au cours du remblayage (de quelque nature qu'il soit) et du forage (rotatif, à sec ou au marteau).

Puis tour à tour, il est question des installations de tir, d'abattage du charbon, du transport des produits et des travailleurs, de préparation du charbon et des minerais en général, des ateliers de mines et du percement des tunnels et des galeries. II faut remarquer que tous ces sujets sont toujours reliés au problème central à savoir la sécurité et l'hygiène industrielles.

Enfin, on arrive à la partie qui me semble la plus intéressante à savoir celle des mesures d'applications pratiques pour la protection individuelle et des méthodes em- 